\title{
Solving the Nurse Scheduling Problem of Private Hospitals in the Philippines using Various Operators for Genetic Algorithm
}

\author{
Rhoda A. Namoco ${ }^{1 *}$ and Ruben G. Salazar ${ }^{2}$ \\ 'Department of Mathematical Sciences, College of Science and Mathematics, University of Science and \\ Technology of Southern Philippines, Lapasan, Cagayan de Oro City, 9000, Philippines; \\ ${ }^{2}$ Agusan National High School, A.D. Curato St., Butuan City, 8600, Philippines; rgsalazar82@gmail.com
}

\begin{abstract}
Objectives: This paper aims to find the most efficient crossover and mutation operators for genetic algorithms in solving the Nurse Scheduling Problem (NSP) for private hospitals in the Philippines. Methods/Statistical Analysis: In this study, different combinations of three crossover and three mutation operators commonly used for Genetic Algorithms (GA) are tested and compared in order to evaluate their efficiency in providing a solution for the NSP. The GA, using the nine different combinations of operators, is then applied to obtain schedules of a particular private hospital in the Philippines. Findings: Results of the study show that the pair two-point crossover and single mutation operators provide a better timetable for nurses at a private hospital in the Philippines in terms of accommodating nurses' preferences and reducing salary costs.Application/Improvements: Private hospitals aiming to find a schedule that respects both the preferences of the nurses and fulfills the objectives of the hospital will find results of this study useful.
\end{abstract}

Keywords: Genetic Algorithm, Nurse Scheduling, Private Hospitals, Philippines

\section{Introduction}

Scheduling is assigning an appropriate number of workers to the jobs during each day of work. Many fields require scheduling such as job scheduling, production scheduling, personnel scheduling and many to mention. Personnel scheduling plays an important task in the companies. It balances the workload of the personnel, departments, and the entire company. Personnel scheduling involves assigning of personnel to times lot and possible location known as time tabling. It is made to maximize the work done needed for the companies. In hospitals, personnel scheduling is applied to assign nurses to their respective departments .

Nurse Scheduling is all about the assignment of shifts and days to nurses. The Nurse Scheduling Problem (NSP) is described as finding a schedule that both respects the constraints of the nurses and fulfills the objectives of the hospital. NSP consists of assigning a work shift to each nurse on each day of a planning period.It can be modeled as a partial constraints satisfaction problem. Various studies have been done for the NSP ${ }^{1-7}$. In the recent years, meta-heuristics such as Tabu search, genetic algorithm, and simulated annealing have been proven to be effective in obtaining good solutions for nurse scheduling problem.

GA have been used in nurse scheduling problem The idea behind genetic algorithm is to breed the fittest solutions in a specific generation. They work with a population of "individuals", each representing a possible solution to a given problem. Each individual is assigned a "fitness score" according to how good a solution is to the problem. Individuals with higher fitness scores are given opportunities to "reproduce" by "cross breeding" with 
other individuals in the population. Genetic Algorithms are powerful general purpose optimization tools which model the principles of evolution. They are often capable of finding globally optimal solutions even in the most complex of search spaces. They operate on a population of coded solutions which are selected according to their quality then used as the basis for a new generation of solutions found by combining (crossover) or altering (mutating) current individuals.

The power of GAs comes from the fact that the technique is robust and can deal successfully with a wide range of problem areas, including those which are difficult for other methods to solve ${ }^{10}$. An NSP model that maintains highest level of service while minimizing hospital-staff costs and fairly distributing overtime among nurses using genetic algorithm was developed in ${ }^{11}$.Results of their study showed that the nurse schedule obtained using their method could save $12 \%$ staffing expenses monthly as compared to existing schedule used by a particular hospital.

In this paper, different combinations of three crossover operators and three mutation operators for genetic algorithms are tested and compared in order to evaluate their efficiency in providing a solution for the NSP.The GA, using the nine different combinations of operators, is then applied to obtain schedules of a particular private hospital in the Philippines. This paper is organized as follows. Section 1 presents an overview of the nurse scheduling problem. The methods used in this study are discussed in section 2. Results and discussion are given in section 3. A brief conclusion is then given in section 4 .

\section{Methodology}

In formulating nurse scheduling problem model, hospital policies are considered. Nurses are required to work 8 hours in a day it either morning, afternoon and night. It usually required working two (2) days for each shift in a week. Private hospitals still follow the statutory regulation for public health workers, and these statutory regulations are then included in the formulation of the NSP model as constraints. Interviews among the hospital administrators and their nurses were conducted in order to determine their preferences, and hence, provide a timetable that is favorable to both parties. The data includes the list of personnel in the eight (8) departments of the hospital and their schedules of days off. Each department is composed of at least 10 nurses. The whole timetable is divided into three shifts: morning, afternoon and night shifts. Morning shifts start at $8 \mathrm{AM}$ and end at $4 \mathrm{PM}$. Afternoon shifts start at $4 \mathrm{PM}$ up to 12 midnight and then followed by the night shifts that start at 12 midnight and end at $8 \mathrm{AM}$ the next day.

The following indices are used in the succeeding discussions.

$\mathrm{I}=\{1,2, \ldots, \mathrm{n}\}$ is the set of all nurses belonging to the same skill level in each department;

$J J=\{1,2,3\}$ is the set of all shifts, that is, morning, afternoon and night shifts;

$\mathrm{K}=\{1,2, \ldots, 7\}$ is the set consisting of days of the week, that is, Mondays thru Sundays;

$\mathrm{L}=\{1,2,3,4\}$ is the set of nurse skill levels (as described in Table 1); and

$\mathrm{N}_{1}, \mathrm{~N}_{2}, \mathrm{~N}_{3}, \mathrm{~N}_{4}$ be the set of nurses with skill level 1,2, 3 , and 4, respectively, as described in Table 1 .

$$
\text { Let } x_{i j k l}=\left\{\begin{array}{l}
1, \text { if nurse } i \text { with skill level } l \text { works on } \\
0, \text { otherwise. }
\end{array}\right.
$$

\section{a shift pattern $j$ on day $k$,}

Based on the results of the interview conducted, the hard constraints considered are as follows. These are based on existing statutory laws, and the preferences of the nurses and the hospital administration.

- Each nurse on duty must work at least 1 shift per day. For each $i \in I, k \in K$, and $l \in L, \sum_{j=1}^{\mathrm{a}} x_{i j k l} \geq \mathbf{1}$.

- Each shift must have at least 1 nurse on duty. For each $j \in J, k \in K$, and $l \in L, \sum_{i=1}^{n} x_{i j k l} \geq \mathbf{1}$.

- No nurse must be assigned to a night shift followed by a morning shift the nextday.

For each $i \in I, k \in K$, and $l \in L$, $x_{i \mathbf{3} k l}+x_{i \mathbf{3}(k+1) l}=\mathbf{1}$.

On the other hand, the soft constraints considered in this study are as follows. These are based on the preferences of the nurses and the hospital administration.

- Each nurse must cover two days of work for every shift each week, that is, nurse $i$ must work two (2) morning shifts, 2 afternoon shifts, and 2 nights shifts For each $i \in I, j \in J$, and $l \in L, \sum_{k=1}^{7} x_{i j k l}=2$. 
- $\quad$ Each nurse must work three (3) feasible shifts in a week, that is, each nurse $i \in I$ must be assigned to a morning shift, afternoon shift and night shift in a

week.
For each $i \in I, j \in J$, and $l \in L, \sum_{k=1}^{7} x_{i j k l}=1$

- Only one day off should take place in a week of work. For each $i \in I$ and $l \in L, \sum_{k=1}^{7} \sum_{j=1}^{3} x_{i j k l}=\mathbf{6}$

- Nurses are not allowed to work during day off.

For each $i \in I, j \in J$, and $l \in L, x_{i j k l}=\mathbf{0}$ for all $k \notin C_{t}$

where $C_{t}$ is the set of all feasible work patterns for shift $j$, and there exists $T$ such sets.

- Only licensed practical and new comer nurses must be assigned on Sunday.

For $k=7$ and for each $i \notin N_{1}$, and $l \in L$, $\sum_{j=1}^{3} x_{i j k l}=\mathbf{0}$.

- Only licensed practical and newcomer nurses and part-time registered nurses should be assigned to night shifts.

For $j=\mathbf{3}$ and for each $i \notin N_{\mathbf{1 u} N_{\mathbf{2}}}$ and $l \in L$, $\sum_{k=1}^{7} x_{i j k l}=0$

The objective of the Nurse Scheduling Problem Minimize $Z=\sum_{m=1}^{6} P_{m}\left(x_{i j k l}\right) S_{i j k l} \begin{aligned} & \text { mize the total salary } \\ & \text { ollows: }\end{aligned}$

where $S_{i j k l}$ is the salary associated to nurse $i$ with skill level $l$ who works on shift $j$ on day $k, P_{m}\left(x_{i j k}\right)$, $m=1,2, \cdots, 6$, correspond to the penalties associated with violations of the above-mentioned soft constraints and are defined as follows:

The following functions represent the penalties imposed whenever each of the abovementioned soft constraints is violated.

For each $\in I, j \in J, k \in K$, and $l \in L$,

$P_{\mathbf{1}}\left(x_{i j k l}\right)=x_{i j k l}(k-2), k>2$;

$P_{\mathbf{2}}\left(x_{i j k l}\right)=x_{i j k l}(3-j), j<\mathbf{3}$;
$P_{\mathrm{a}}\left(x_{i j k l}\right)=x_{i j k l}(k-1), k>1$;

$P_{\mathbf{4}}\left(x_{i j k l}\right)=\mathbf{4} x_{i j k l}$;

$P_{5}\left(x_{i j k l}\right)=x_{i j k l}(l-1)$, where $l=2,3,4$ is the skill level of nurse $i \in I$;

$P_{\mathbf{6}}\left(x_{i j k}\right)=x_{i j k}(l-2)$, where $l=3,4$ is the skill level of nurse $i \in I$.

Correspondingly, violations of the soft constraints in each of the resulting timetable is computed using the penalty function

$$
\begin{aligned}
& P\left(x_{i j k l}\right)=\sum_{l=\mathbf{1}}^{\mathbf{4}} \sum_{k=\mathbf{1}}^{\mathbf{7}} \sum_{j=\mathbf{1}}^{\mathbf{3}} \sum_{i=\mathbf{1}}^{n} \mathbb{\mathbb { }}\left(P \beth_{\mathbf{1}}\left(x_{i j k l}\right)+P_{\mathbf{2}}\left(x_{i j k l}\right)\right. \\
& \left.+P_{\mathbf{3}}\left(x_{i j k l}\right)+P_{\mathbf{4}}\left(x_{i j k l}\right)+P_{\mathbf{5}}\left(x_{i j k l}\right)+P_{\mathbf{6}}\left(x_{i j k l}\right)\right) .
\end{aligned}
$$

In order to evaluate the quality of solutions obtained by any pairs of operators used to obtain timetables, the fitness function is defined by

$f(x)=\frac{1}{1+P\left(x_{i j k l}\right)}$

The timetable with a higher fitness value is then selected as parents for the next generation. It means that the timetable with fewer violations of the soft constraints is considered as the fitter timetable. Correspondingly, the more the violations committed, the smaller the fitness value become. When there are no soft constraints violated, the fitness value becomes 1 .

This study applies Genetic Algorithm (GA) to solve the nurse scheduling problem for private hospitals in the Philippines. The basic techniques of GAs are designed to simulate processes in natural systems necessary for evolution. Associated with GA are the genetic operators. These operators are the ones responsible for creating wide range of solutions. The two main kinds of operators responsible for breeding or producing individuals are the recombination (crossover) and mutation. In this study, 3 crossover operators and 3 mutation operators are considered and compared in order to determine which pair of operators will give the best timetable for nurses in 
private hospitals of the Philippines. These operators are as shown in Table 1.

Table 1. Crossover and mutation operators used

\begin{tabular}{ll}
\hline Crossover Operators $(\mathrm{C})$ & Mutation Operators $\left(\mathrm{M}_{\mathrm{o}}\right)$ \\
\hline Single-pointCrossover $\left(\mathrm{C}_{\mathrm{o}} 1\right)$ & $\begin{array}{l}\text { Uniform Mutation Operator } \\
\left(\mathrm{M}_{\mathrm{o}} 1\right)\end{array}$ \\
Two-point Crossover $\left(\mathrm{C}_{\mathrm{o}} 2\right)$ & $\begin{array}{l}\text { Single Mutation Operator } \\
\left(\mathrm{M}_{\mathrm{o}} 2\right)\end{array}$ \\
Uniform Crossover $\left(\mathrm{C}_{\mathrm{o}} 3\right)$ & $\begin{array}{l}\text { Boundary Mutation Operator } \\
\left(\mathrm{M}_{0} 3\right)\end{array}$ \\
\hline
\end{tabular}

Crossover operators produce offspring by recombining the information from two parents. It is the major exploratory mechanism of the genetic algorithm. Many crossover techniques exist for organisms which use different data structures to store themselves. The singlepoint cross over $\left(\mathrm{C}_{\mathrm{o}} 1\right)$ randomly selects a crossover point within a chromosome then interchanges the two parent chromosomes at this point to produce two new offspring Figure 1.
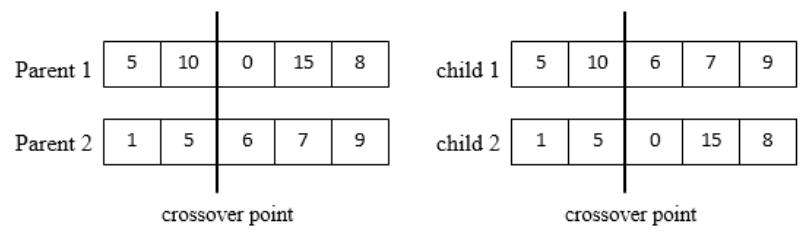

Figure 1. Single-point crossover.

The two-point crossover $\left(\mathrm{C}_{\mathrm{o}} 2\right)$ randomly selects two crossover points within chromosomes then interchanges the two parent chromosomes between these points to produce two new offspring. An illustration of this method is shown in Figure 2.
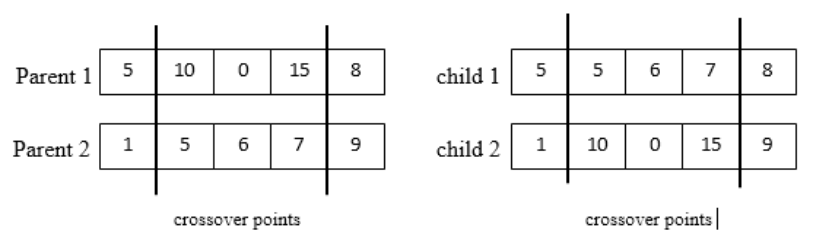

Figure 2. Two-point crossover.

Uniform Crossover ( $\mathrm{C}_{\mathrm{o}} 3$ ) decides (with some probability - referred to as the mixing ratio) which parent will contribute each of the gene values in the offspring chromosomes. This allows the parent chromosomes to be mixed at the gene level rather than the segment level (as with one and two-point crossover). The usual mixing ratio is at $50 \%$, so approximately, half of the gene from the offspring will come from Parent 1 and the other half from Parent 2. This method is demonstrated in Figure 3.

\begin{tabular}{|c|c|c|c|c|c|c|c|c|c|c|c|}
\hline Parent 1 & 5 & 10 & 0 & 15 & 8 & \multirow[t]{2}{*}{ child } & 5 & 3 & 6 & 15 & 8 \\
\hline Parent 2 & 1 & 3 & 6 & 7 & 9 & & 1 & 2 & 2 & 1 & 1 \\
\hline & 1 & 2 & 2 & 1 & 1 & & & & & & \\
\hline
\end{tabular}

Figure 3. Uniform crossover.

On the other hand, mutation operators used in this study include uniform mutation operator which is a twostep process. Firstly, the algorithm selects a fraction of the vector entries of an individual for mutation, where each entry has the same probability as the mutation rate of being mutated. In the second step, the algorithm replaces each selected entry by a random number selected uniformly from the range for that entry. Another mutation operator considered in this study is the Single Mutation Operator $\left(\mathrm{M}_{\mathrm{o}} 2\right)$. This method randomly selects a single point from a solution and alters its value. This gives the population diversity which ensures that each solution would not be identical thus avoiding premature convergence. This method is shown in Figure 4.

Before

\begin{tabular}{|l|l|l|l|l|}
\hline 5 & 3 & 6 & 10 & 8 \\
\hline
\end{tabular}

Mutation point 3

After

\begin{tabular}{|l|l|l|l|l|}
\hline 5 & 3 & 7 & 10 & 8 \\
\hline
\end{tabular}

Figure 4. Single mutation operator.

Boundary Mutation Operator $\left(\mathrm{M}_{0} 3\right)$ is also considered in this study. This operator replaces the value of the chosen gene with either the upper or lower bound for that gene. Example from the vector entities of individual ranging from 1 to 10 , where 1 is the lower bound and 10 is the upper bound. Algorithm replaces randomly the gene entity by 1 or 10 with equal probability as the mutation rate. Figure 5 illustrates the boundary mutation method. 
Before

\begin{tabular}{|l|l|l|l|l|}
\hline 5 & 3 & 7 & 4 & 8 \\
\hline
\end{tabular}

Mutation point 3 and 5

After

\begin{tabular}{|l|l|l|l|l|}
\hline 5 & 3 & 1 & 4 & 10 \\
\hline
\end{tabular}

Figure 5. Boundary mutation operator.

The resulting timetables obtained using the different combinations of these crossover and mutation operators are then evaluated using the fitness function. The highest fitness evaluation value is then considered as the most efficient genetic operator for NSP.

The morning, afternoon and night time salary rates for nurses employed in a private hospital as well as the nurse timetables being implemented by the hospital were also gathered during the interview. These are shown in Tables 2 and 3. It can be seen from the tables that salary of nurses varies according to their skill level, the day of the week and the shift they are assigned to.
In this study, the same crossover point is used for the different operators considered, namely, $3^{\text {rd }}$ crossover point for single crossover; in between the $1^{\text {st }}$ and $5^{\text {th }}$ crossover point for the two-point crossover; and for the uniform crossover, it is set randomly to any crossover point with size of 3 points ( 3 different days). For mutation operators, uniform mutation is set to $5 \%$, single mutation mutates only one shift every generation and boundary mutation is set to $2 \%$. The algorithm has been tested using 1000 generations.

\section{Results and Discussion}

Figure 6 shows a sample of the Graphical User Interface (GUI) of the nurse scheduler used in this study for testing the efficiency of the genetic operators. Figure 7 shows a sample of how parameters are set in order to make a nurse schedule for 15 nurses in the Operating room (OR) department with 6 nurses of skill level 1, 3 nurses of level 2, 3 nurses of level 3, and 3 nurses of level 4, assigned to three different shifts for a week.

Table 2. Salary rates of nurses for morning and afternoon shifts

\begin{tabular}{|l|c|c|c|c|c|c|c|c|c|c|}
\hline \multirow{2}{*}{ Nurse Classification } & \multirow{2}{*}{$\begin{array}{c}\text { Skill } \\
\text { Level }\end{array}$} & \multicolumn{6}{|c|}{ Daytime Salary of Nurses (in Philippine peso, PHP. 1USD = 47PHP) } \\
\cline { 3 - 9 } & & Monday & Tuesday & Wednesday & Thursday & Friday & \multicolumn{2}{|c|}{ Saturday } & \multicolumn{2}{|c|}{ Sunday } \\
\hline $\begin{array}{l}\text { Clinical nurse } \\
\text { specialist }\end{array}$ & 4 & 692 & 692 & 692 & 692 & 692 & 692 & 899 \\
\hline $\begin{array}{l}\text { Regular registered } \\
\text { nurse }\end{array}$ & 3 & 576 & 576 & 576 & 576 & 576 & 576 & 749 \\
\hline $\begin{array}{l}\text { Part-time registered } \\
\text { nurse }\end{array}$ & 2 & 461 & 461 & 461 & 461 & 461 & 461 & 599 \\
\hline $\begin{array}{l}\text { Licensed practical } \\
\text { and newcomer nurse }\end{array}$ & 1 & 384 & 384 & 384 & 384 & 384 & 384 & 499 \\
\hline
\end{tabular}

Table 3. Salary rates of nurses for night shift

\begin{tabular}{|l|c|c|c|c|c|c|c|c|c|}
\hline \multicolumn{1}{|c|}{ Nurse Classification } & \multirow{2}{*}{$\begin{array}{c}\text { Skill Level } \\
\text { Monday }\end{array}$} & \multicolumn{6}{c|}{ Nighttime Salary of Nurses (in Philippine peso, PHP. 1USD = 47PHP) } \\
\cline { 3 - 11 } & Tuesday & Wednesday & Thursday & Friday & Saturday & Sunday & \\
\hline Clinical nurse specialist & 4 & 761 & 761 & 761 & 761 & 761 & 761 & 988 \\
\hline Regular registered nurse & 3 & 633 & 633 & 633 & 633 & 633 & 633 & 823 \\
\hline Part-time registered nurse & 2 & 507 & 507 & 507 & 507 & 507 & 507 & 658 \\
\hline $\begin{array}{l}\text { License practical and } \\
\text { newcomer nurse }\end{array}$ & 1 & 422 & 422 & 422 & 422 & 422 & 422 & 548 \\
\hline
\end{tabular}




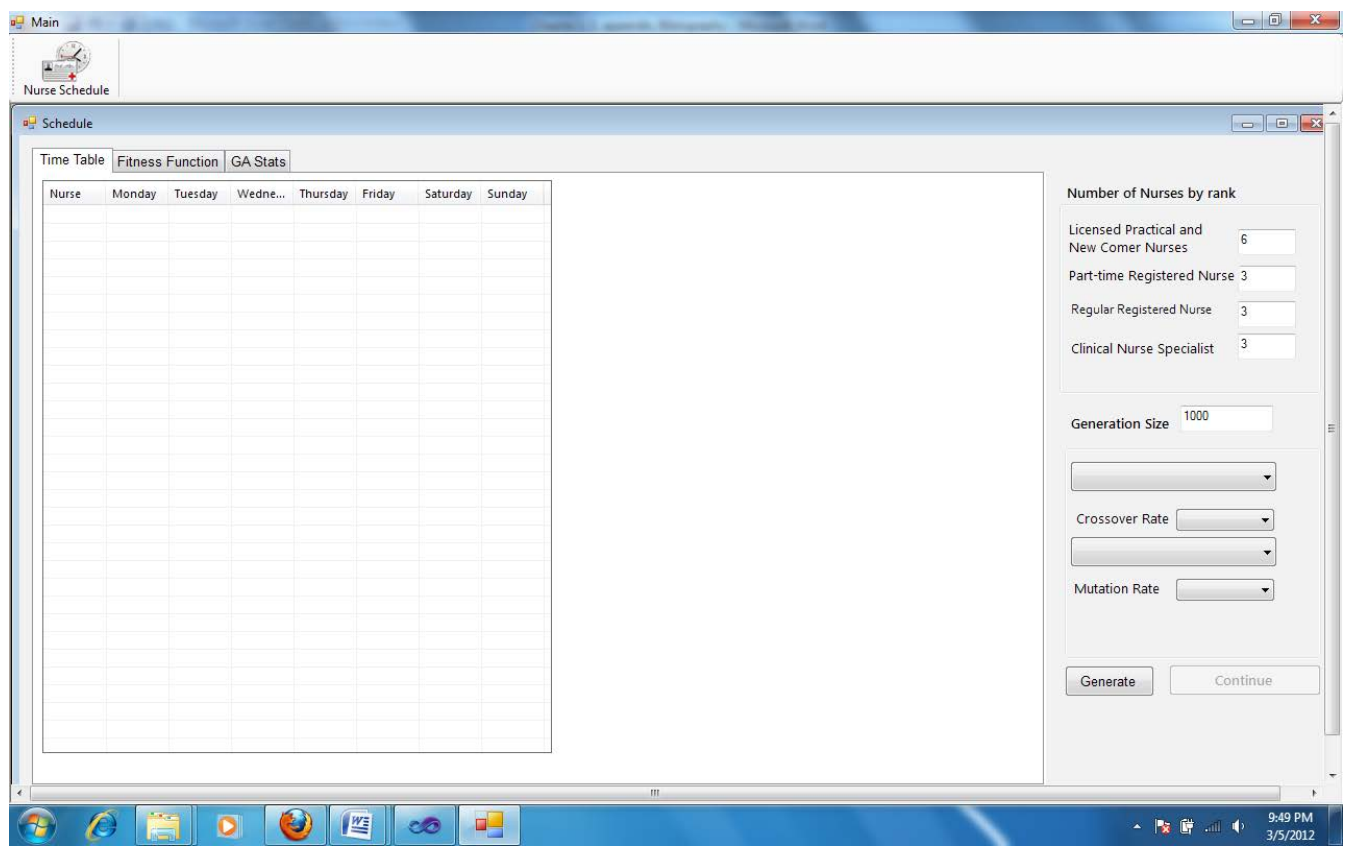

Figure 6. Sample of GUI of nurse scheduler system used in this study.

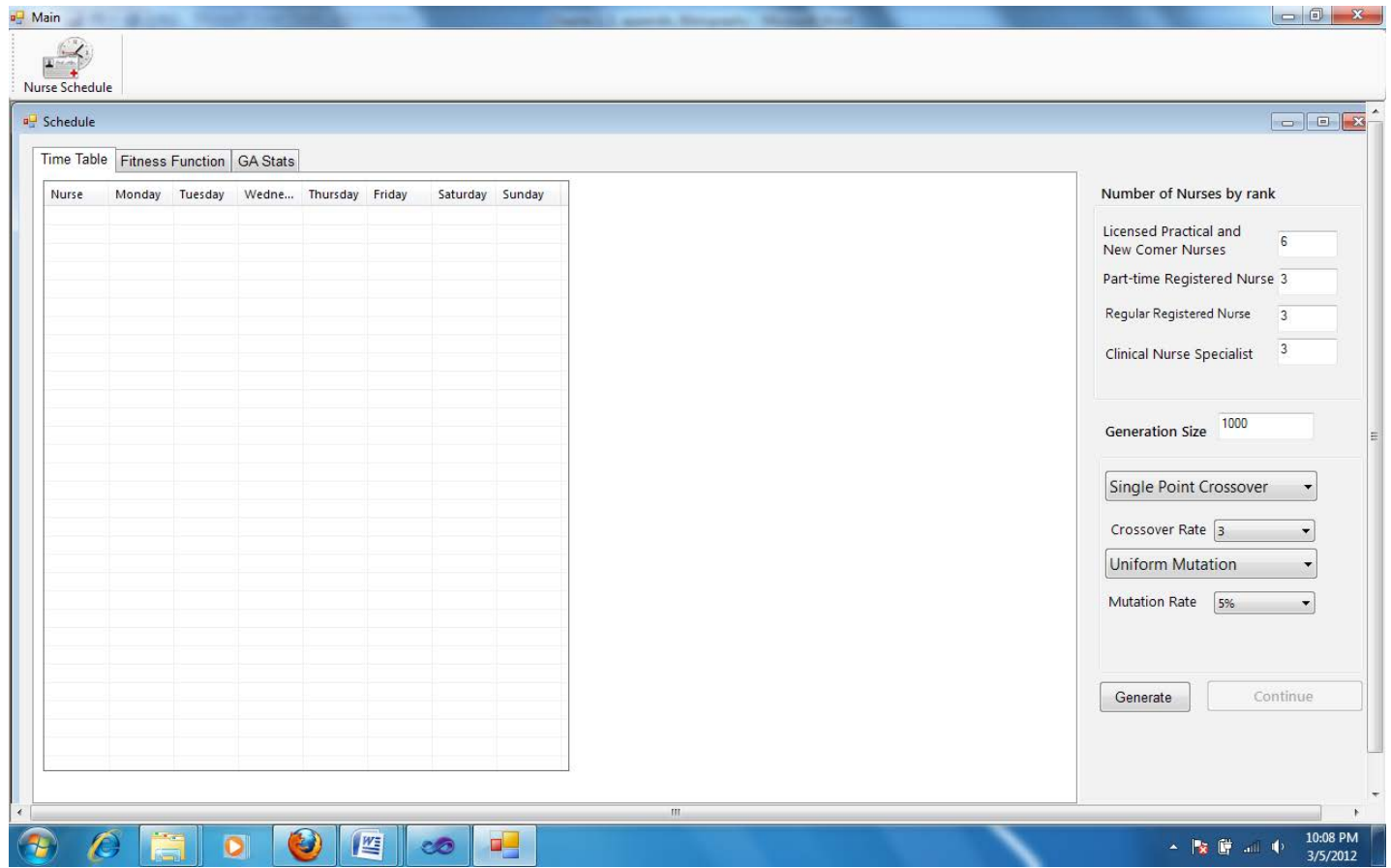

Figure 7. Sample showing how to set the parameters using the nurse scheduler.

Figures 8 and 9 show the timetable (individual) generated by the proposed nurse scheduler. In the tables, Nurse 1 corresponds to a nurse with skill level 1, Nurse 2 to a nurse with skill level 2, and so on. The time table shows that there are six nurses with skill level 1, three nurses with skill level 2, three nurses with skill level 3, and three nurses with skill level 4 assigned in a week schedule. It can be seen that every nurse is assigned to only one shift per day. The time table also reflects the requirement that no nurse is assigned to a night followed by a morning shift the next day. 


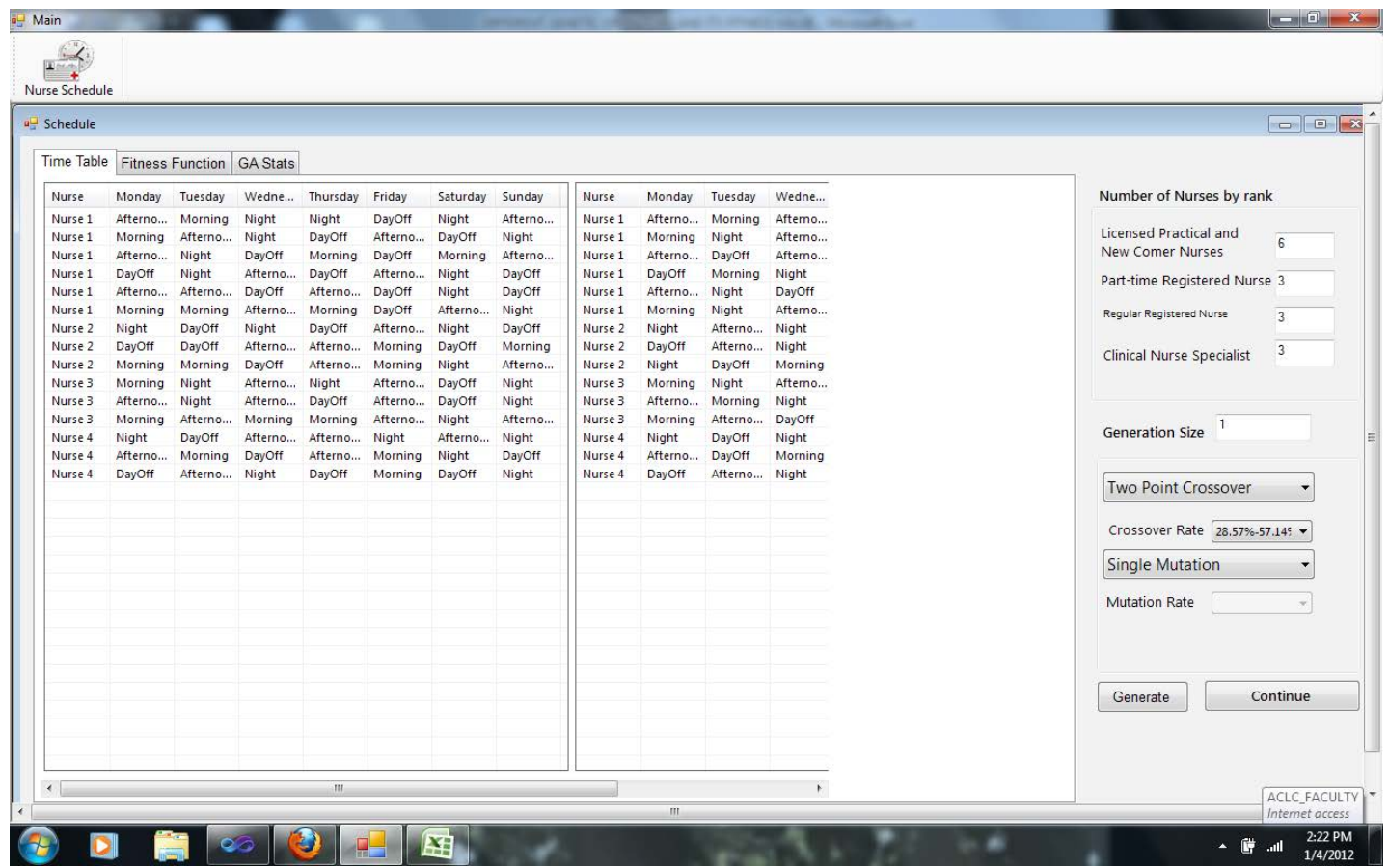

Figure 8. Sample nurse timetable (parent 1) generated by the automated nurse scheduler.

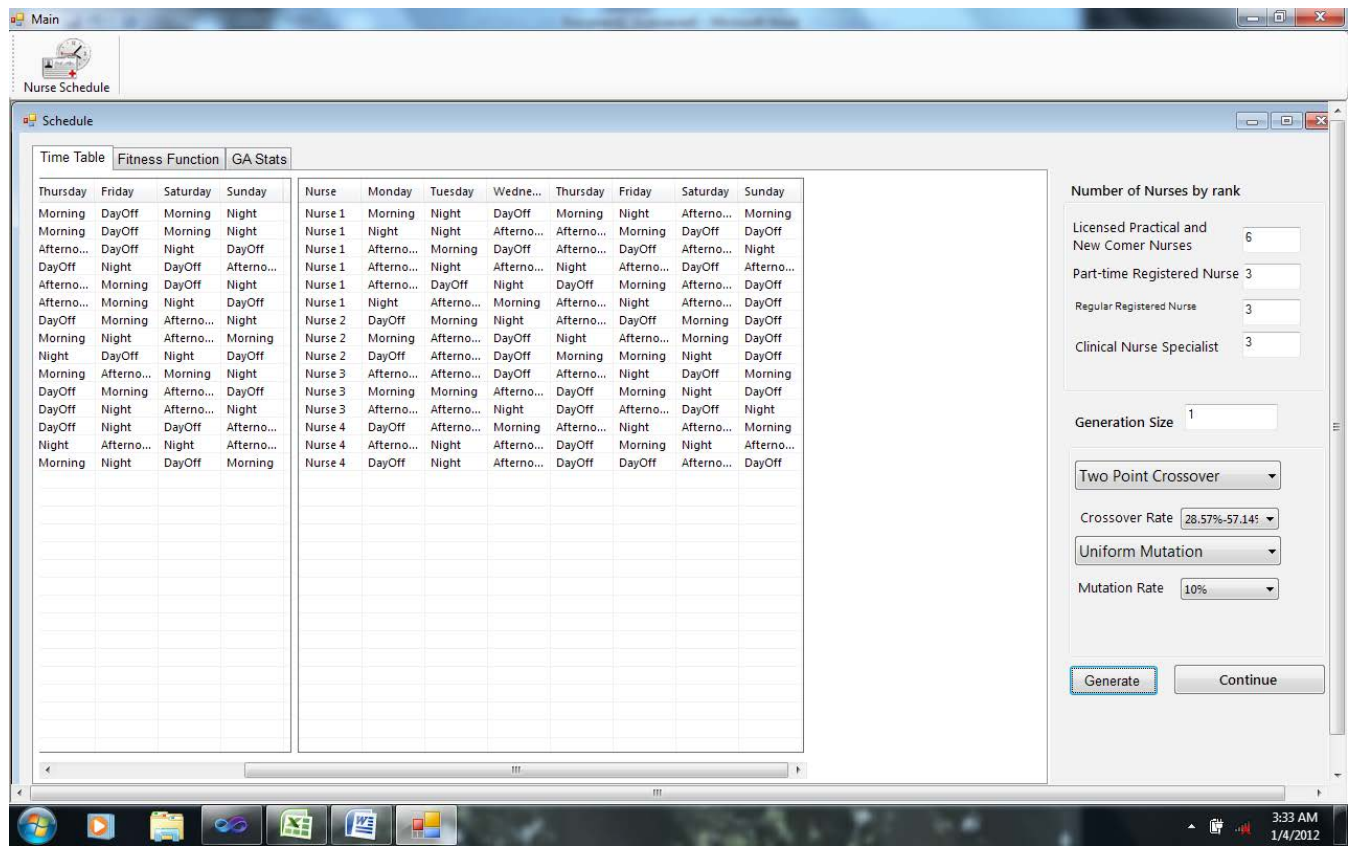

Figure 9. Sample nurse timetable (parent 2) generated by the automated nurse scheduler.

It can be seen that the first timetable (Parent 1), shown in Figure 3, satisfies the hard constraints. After generating the first timetable, another feasible timetable is generated, and this is used as parent 2 shown in Figure 4. These two timetables are then used to produce children by applying the different genetic operators.
In order to evaluate the performance of the different genetic operators, fitness evaluation values of the resulting timetables for the nurse scheduling problem with various crossover and mutation operators obtained have been computed and compared using the fitness evaluation function (1). Figure 10 shows that soft constraint \#1 is 


\begin{tabular}{|c|c|c|c|c|c|c|c|}
\hline Nurse & Monday & Tuesday & Wedne... & Thursday & Friday & Saturday & Sunday \\
\hline Nurse 1 & Afterno... & Morning & Night & Night & DayOff & Night & Afterno... \\
\hline Nurse 1 & Morning & Afterno... & Night & Dayoff & Afterno... & DayOff & Night \\
\hline Nurse 1 & Afterno... & Night & DayOtf & Morning & DayOff & Morning & Afterno... \\
\hline Nurse 1 & DayOff & Night & Afterno... & DayOff & Afterno... & Night & DayOff \\
\hline Nurse 1 & Afterno... & Afterno... & Dayoff & Afterno... & Dayoff & Night & Dayoft \\
\hline Nurse 1 & Morning & Morning & Afterno... & Morning & Dayoff & Afterno... & Night \\
\hline Nurse 2 & Night & DayOff & Night & DayOff & Afterno... & Night & DayOff \\
\hline Nurse 2 & DayOtt & Dayoft & Afterno... & Afterno... & Morning & Dayott & Morning \\
\hline Nurse 2 & Morning & Morning & DayOff & Afterno... & Morning & Night & Afterno... \\
\hline Nurse 3 & Morning & Night & Afterno... & Night & Afterno... & DayOff & Night \\
\hline Nurse 3 & Afterno... & Night & Afterno... & Dayotf & Afterno... & Dayotf & Night \\
\hline Nurse 3 & Morning & Afterno... & Morning & Morning & Afterno... & Night & Afterno... \\
\hline Nurse 4 & Night & DayOff & Afterno... & Afterno... & Night & Afterno... & Night \\
\hline Nurse 4 & Afterno... & Morning & DayOtf & Afterno... & Morning & Night & Dayotf \\
\hline Nurse 4 & DayOff & Afterno... & Night & DayOff & Morning & DayOff & Night \\
\hline
\end{tabular}

Figure 10. Timetable showing a nurse schedule violating soft constraint \#1.

\begin{tabular}{||lllllllll||}
\hline Nurse & Monday & Tuesday & Wedne... & Thursday & Friday & Saturday & Sunday \\
Nurse 1 & Afterno... & Morning & Night & Night & DayOff & Night & Afterno... \\
Nurse 1 & Morning & Afterno... & Night & DayOff & Afterno... & DayOff & Night \\
Nurse 1 & Afterno... & Niaht & Danoff & Morning & DayOff & Morning & Afterno... \\
Nurse 1 & DayOff & Night & Afterno... & DayOff & Afterno... & Night & DayOff \\
Nurse 1 & Afterno... & Afterno... & Afte... & Afterno... & DayOff & Night & DayOff \\
Nurse 1 & Morning & Morning & Afterno... & Morning & DayOff & Afterno... & Night \\
Nurse 2 & Night & DayOff & Night & DayOff & Afterno... & Night & DayOff \\
Nurse 2 & DayOff & DayOff & Afterno... & Afterno... & Morning & DayOff & Morning \\
Nurse 2 & Morning & Morning & DayOff & Afterno... & Morning & Night & Afterno... \\
Nurse 3 & Morning & Night & Afterno... & Night & Afterno... & DayOff & Night \\
Nurse 3 & Afterno... & Night & Afterno... & DayOff & Afterno... & DayOff & Night \\
Nurse 3 & Morning & Afterno... & Morning & Morning & Afterno... & Night & Afterno... \\
Nurse 4 & Night & DayOff & Afterno... & Afterno... & Night & Afterno... & Night \\
Nurse 4 & Afterno... & Morning & DayOff & Afterno... & Morning & Night & DayOff \\
Nurse 4 & DayOff & Afterno... & Night & DayOff & Morning & DayOff & Night \\
& & & & & & & & \\
\hline
\end{tabular}

Figure 11. Timetable showing a nurse Schedule violating soft constraint \#2.

\begin{tabular}{||ll|l|l|l|l|l|l|}
\hline Nurse & Monday & Tuesday & Wedne... & Thursday & Friday & Saturday & Sunday \\
Nurse 1 & Afterno... & Morning & Night & Night & DayOff & Night & Afterno... \\
Nurse 1 & Morning & Afterno... & Night & DayOff & Afterno... & DayOff & Night \\
Nurse 1 & Afterno... & Night & DayOff & Morning & DayOff & Morning & Afterno... \\
Nurse 1 & DayOff & Night & Afterno... & DayOff & Afterno... & Night & DayOff \\
Nurse 1 & Afterno... & Afterno... & DayOff & Afterno... & DayOff & Night & DayOff \\
Nurse 1 & Morning & Morning & Afterno... & Morning & DayOff & Afterno... & Night \\
Nurse 2 & Night & DayOff & Night & DayOff & Afterno... & Night & DayOff \\
Nurse 2 & DayOff & DayOff & Afterno... & Afterno... & Morning & DayOff & Morning \\
Nurse 2 & Morning & Morning & DayOff & Afterno... & Morning & Night & Afterno... \\
Nurse 3 & Morning & Night & Afterno... & Night & Afterno... & DayOff & Night \\
Nurse 3 & Afterno... & Night & Afterno... & DayOff & Afterno... & DayOff & Night \\
Nurse 3 & Morning & Afterno... & Morning & Morning & Afterno... & Night & Afterno... \\
Nurse 4 & Night & DayOff & Afterno... & Afterno... & Night & Afterno... & Night \\
Nurse 4 & Afterno... & Morning & DayOff & Afterno... & Morning & Night & DayOff \\
Nurse 4 & DayOff & Afterno... & Night & DayOff & Morning & DayOff & Night \\
\hline
\end{tabular}

Figure 12. Timetable showing nurse schedule violating soft constraint \#3. 


\begin{tabular}{|c|c|c|c|c|c|c|c|}
\hline Nurse & Monday & Tuesday & Wedne... & Thursday & Friday & Saturday & Sunday \\
\hline Nurse 1 & Afterno... & Morning & Night & Night & DayOff & Night & Afterno... \\
\hline Nurse 1 & Morning & Afterno... & Night & DayOff & Afterno... & DayOff & Night \\
\hline Nurse 1 & Afterno... & Night & DayOff & Morning & Dayoff & Morning & Afterno... \\
\hline Nurse 1 & DayOff & Night & Afterno... & DayOff & Afterno... & Night & DayOff \\
\hline Nurse 1 & Afterno... & Afterno... & Dayotf & Afterno... & Dayoff & Night & Dayoff \\
\hline Nurse 1 & Morning & Morning & Afterno... & Morning & DayOff & Afterno... & Night \\
\hline Nurse 2 & Night & DayOff & Night & DayOff & Afterno... & Night & DayOff \\
\hline Nurse 2 & Dayoff & Dayoff & Afterno... & Afterno... & Morning & DayOff & Morning \\
\hline Nurse 2 & Morning & Morning & DayOff & Afterno... & Morning & Night & Afterno... \\
\hline Nurse 3 & Morning & Night & Afterno... & Night & Afterno... & DayOff & Night \\
\hline Nurse 3 & Afterno & Niaht & Afterno & Davoft & Afternom & Davoft & Niaht \\
\hline Nurse 3 & Morning & Afterno... & Morning & Morning & Afterno... & Night & Afterno... \\
\hline Nurse 4 & Tirgnt & vayort & Atremo... & Atremo... & Tignt & Aarternow... & Firgit \\
\hline Nurse 4 & Afterno... & Morning & DayOff & Afterno... & Morning & Night & DayOtf \\
\hline Nurse 4 & DayOff & Afterno... & Night & Dayoff & Morning & DayOff & Night \\
\hline
\end{tabular}

Figure 13. Timetable showing nurse schedule violating soft constraint \#4.

violated by parent 1 (earlier shown in Figure 3).It shows that first nurse level 1 is scheduled to work for 3 night shifts in a week. This schedule violates soft constraint \#1. This means that the first nurse of level 1 (labeled Nurse 1) gets a penalty of 1 . Nurse 3 also gets a penalty of 1 .

Figure 11 shows that fourth nurse level 1 has no morning shift schedule for the week considered in this timetable. Hence, this timetable violates soft constraints $\# 2$. Correspondingly, it gets a penalty of 1 due to not having a morning shift.

It can be seen from Figure 12 that the schedule of the fifth nurse level 1 violates soft constraint \#3, that is, that there should be at least 1 day off in a week. The timetable shows that there are 3 day offs of fifth nurse level 1 . Hence a penalty of 2 . Figure 13 , on the other, shows that the timetable violates soft constraint \#4 and is penalized with 4 units, since the third nurse of level 3 has no day off.
Soft constraint \#5 requires that only nurse level 1 will work during Sunday. Figure 14 shows that first nurse level 4 works on Sunday night; hence the solution is penalized by a unit of 3 .

Figure 15 shows that this timetable violates soft constraint \#6, which corresponds to a penalty of 4 units since the third nurse level 4 was scheduled to work on a night shift twice in this timetable.

Using the fitness evaluation function (1), timetable labeled parent 1 has a fitness value of 0.014706 while timetable parent 2 has a fitness value of 0.02 .

Figure 16 shows timetable generated using the parameters single-point crossover $\left(\mathrm{C}_{\mathrm{o}} 1\right)$ and uniform mutation $\left(M_{0} 1\right)$ operators. In this experiment, the $3^{\text {rd }}$ crossover point is used in the $\mathrm{C}_{\mathrm{o}} 1$ operator and $5 \%$ mutation rate for $M_{0} 1$ operator in the first generation. The first part of the timetable is inherited from the first

\begin{tabular}{|c|c|c|c|c|c|c|c|}
\hline Nurse & Monday & Tuesday & Wedne... & Thursday & Friday & Saturday & Sunday \\
\hline Nurse 1 & Afterno... & Morning & Night & Night & DayOff & Night & Afterno... \\
\hline Nurse 1 & Morning & Afterno... & Night & Dayoff & Afterno... & Dayoff & Night \\
\hline Nurse 1 & Afterno... & Night & DayOff & Morning & DayOff & Morning & Afterno... \\
\hline Nurse 1 & DayOff & Night & Afterno... & DayOff & Afterno... & Night & DayOff \\
\hline Nurse 1 & Afterno... & Afterno... & DayOtf & Afterno... & DayOff & Night & Dayoff \\
\hline Nurse 1 & Morning & Morning & Afterno... & Morning & DayOff & Afterno... & Night \\
\hline Nurse 2 & Night & DayOff & Night & DayOff & Afterno... & Night & DayOff \\
\hline Nurse 2 & Dayoff & Dayoft & Afterno... & Afterno... & Morning & DayOtf & Morning \\
\hline Nurse 2 & Morning & Morning & DayOff & Afterno... & Morning & Night & Afterno... \\
\hline Nurse 3 & Morning & Night & Afterno... & Night & Afterno... & DayOff & Night \\
\hline Nurse 3 & Afterno... & Night & Afterno... & DayOff & Afterno... & DayOff & Night \\
\hline Nurse 3 & Morning & Afterno... & Morning & Morning & Afterno... & Night & Afterno... \\
\hline Nurse 4 & Night & DayOff & Afterno... & Afterno... & Night & Afterno... & Night \\
\hline Nurse 4 & Afterno... & Morning & DayOff & Afterno... & Morning & Night & Dayotf \\
\hline Nurse 4 & DayOff & Afterno... & Night & DayOff & Morning & DayOff & Night \\
\hline
\end{tabular}

Figure 14. Timetable showing nurse schedule violating soft constraint\# 5 . 


\begin{tabular}{||lllllllll||}
\hline Nurse & Monday & Tuesday & Wedne... & Thursday & Friday & Saturday & Sunday \\
Nurse 1 & Afterno... & Morning & Night & Night & DayOff & Night & Afterno... \\
Nurse 1 & Morning & Afterno... & Night & DayOff & Afterno... & DayOff & Night \\
Nurse 1 & Afterno... & Night & DayOff & Morning & DayOff & Morning & Afterno... \\
Nurse 1 & DayOff & Night & Afterno... & DayOff & Afterno... & Night & DayOff \\
Nurse 1 & Afterno... & Afterno... & DayOff & Afterno... & DayOff & Night & DayOff \\
Nurse 1 & Morning & Morning & Afterno... & Morning & DayOff & Afterno... & Night \\
Nurse 2 & Night & DayOff & Night & DayOff & Afterno... & Night & DayOff \\
Nurse 2 & DayOff & DayOff & Afterno... & Afterno... & Morning & DayOff & Morning \\
Nurse 2 & Morning & Morning & DayOff & Afterno... & Morning & Night & Afterno... \\
Nurse 3 & Morning & Night & Afterno... & Night & Afterno... & DayOff & Night \\
Nurse 3 & Afterno... & Night & Afterno... & DayOff & Afterno... & DayOff & Night \\
Nurse 3 & Morning & Afterno... & Morning & Morning & Afterno... & Night & Afterno... \\
Nurse 4 & Night & DayOff & Afterno... & Afterno... & Night & Afterno... & Night \\
Nurse 4 & Afterno... & Morning & Davoff & Afterno... & Morning & Night & DayOff \\
Nurse 4 & DayOff & Afterno... & Night & DayOff & Morning & DayOff & Dight \\
\hline
\end{tabular}

Figure 15. Timetable showing nurse schedule violating soft constraint \#6.

\begin{tabular}{|c|c|c|c|c|c|c|c|}
\hline Nurse & Monday & Tuesday & Wedne... & Thursday & Friday & Saturday & Sunday \\
\hline Nurse1 & Afterno... & Morning & DayOff & Morning & Night & Afterno... & Morning \\
\hline Nurse1 & Morning & Afterno... & Night & Afterno... & Morning & DayOff & DayOff \\
\hline Nurse1 & Afterno... & Night & DayOff & Afterno... & DayOff & Afterno... & Night \\
\hline Nurse1 & DayOff & Night & Afterno... & Night & Afterno... & DayOff & Afterno... \\
\hline Nurse1 & Afterno... & Afterno... & DayOff & DayOff & Morning & Afterno... & DayOff \\
\hline Nurse1 & Morning & Morning & Afterno... & Afterno... & Night & Afterno... & Afterno... \\
\hline Nurse2 & Night & DayOff & Night & Afterno... & DayOff & Morning & DayOff \\
\hline Nurse2 & DayOff & DayOff & Afterno... & Night & Afterno... & Morning & DayOff \\
\hline Nurse2 & Morning & Morning & DayOff & Morning & Morning & Night & DayOff \\
\hline Nurse 3 & Morning & Night & Afterno... & Afterno... & Night & DayOff & Morning \\
\hline Nurse3 & Afterno... & Night & Afterno... & DayOff & Morning & Night & DayOff \\
\hline Nurse 3 & Morning & Afterno... & Morning & DayOff & Night & DayOff & Night \\
\hline Nurse 4 & Afterno... & DayOff & Afterno... & Afterno... & Night & Afterno... & Morning \\
\hline Nurse 4 & Afterno... & Morning & DayOff & DayOff & Morning & Night & Afterno... \\
\hline Nurse4 & DayOff & Afterno... & Morning & DayOff & DayOff & Afterno... & DayOff \\
\hline
\end{tabular}

Figure 16. Sample timetable generated using single-point crossover and uniform mutation operators.

parent and the other part is from the second parent. The highlighted parts (afternoon, day off, morning, night and afternoon) are the mutated parts of the timetable from its parents.

The timetable shown in Figure 17 illustrates the procedure when using the parameters single point crossover point $\left(\mathrm{C}_{\mathrm{o}} 1\right)$ and single mutation $\left(\mathrm{M}_{\mathrm{o}} 2\right)$ at first generation. Setting the same crossover point for single crossover, the first part of the timetable inherits the schedule from the first parent and the other part is from the second parent. It can be seen that there is a mutated part from the second parent, i.e., from morning shift it becomes night shift.

Figure 18 presents the timetable generated by the first generation using the parameters single-point crossover and boundary mutation. Setting the same crossover point for single crossover $\left(\mathrm{C}_{\mathrm{o}} 1\right)$ and the mutation rate for boundary mutation $\left(\mathrm{M}_{\mathrm{o}} 3\right)$ at $2 \%$, the behavior of boundary is to change the genes by upper value which is morning and lower value is night. Hence, the night shift from the first parent becomes morning shift and in the second parent, the morning becomes night. 


\begin{tabular}{|c|c|c|c|c|c|c|c|}
\hline Nurse & Monday & Tuesday & Wedne... & Thursday & Friday & Saturday & Sunday \\
\hline Nurse1 & Afterno... & Morning & Night & Night & Night & Afterno... & Morning \\
\hline Nurse1 & Morning & Afterno... & Night & Afterno... & Morning & DayOtf & DayOff \\
\hline Nurse1 & Afterno... & Night & DayOff & Afterno... & DayOff & Afterno... & Night \\
\hline Nurse1 & DayOff & Night & Afterno... & Night & Afterno... & DayOff & Afterno... \\
\hline Nurse1 & Afterno... & Afterno... & Dayoff & DayOff & Morning & Afterno... & DayOff \\
\hline Nurse1 & Morning & Morning & Afterno... & Afterno... & Night & Afterno... & DayOff \\
\hline Nurse2 & Night & Dayoff & Night & Afterno... & Dayoff & Morning & Dayoff \\
\hline Nurse2 & DayOff & DayOff & Afterno... & Night & Afterno... & Morning & DayOff \\
\hline Nurse2 & Morning & Morning & Dayoff & Morning & Morning & Night & Dayoff \\
\hline Nurse3 & Morning & Night & Afterno... & Afterno... & Night & Dayoff & Morning \\
\hline Nurse 3 & Afterno... & Night & Afterno... & DayOff & Morning & Night & DayOff \\
\hline Nurse 3 & Morning & Afterno... & Morning & DayOff & Afterno... & DayOff & Night \\
\hline Nurse4 & Night & DayOff & Afterno... & Afterno... & Night & Afterno... & Morning \\
\hline Nurse 4 & Afterno... & Morning & Dayoff & DayOff & Morning & Night & Afterno... \\
\hline Nurse4 & DayOff & Afterno... & Night & DayOff & Dayoff & Afterno... & DayOff \\
\hline
\end{tabular}

Figure 17. Sample timetable generated using single-point crossover and single mutation operators.

\begin{tabular}{|c|c|c|c|c|c|c|c|}
\hline Nurse & Monday & Tuesday & Wedne... & Thursday & Friday & Saturday & Sunday \\
\hline Nurse1 & Afterno... & Morning & Morning & Morning & Night & Afterno... & Morning \\
\hline Nurse1 & Morning & Afterno... & Night & Afterno... & Morning & DayOff & DayOff \\
\hline Nurse1 & Afterno... & Night & DayOff & Afterno... & DayOff & Afterno... & Night \\
\hline Nurse1 & DayOff & Night & Afterno... & Night & Afterno... & DayOff & Afterno... \\
\hline Nurse1 & Afterno... & Afterno... & DayOff & DayOff & Morning & Afterno... & DayOff \\
\hline Nurse1 & Morning & Morning & Afterno... & Afterno... & Night & Afterno... & Dayoff \\
\hline Nurse2 & Night & DayOff & Night & Afterno... & DayOff & Morning & DayOff \\
\hline Nurse2 & DayOff & DayOff & Afterno... & Night & Afterno... & Morning & DayOff \\
\hline Nurse2 & Morning & Morning & DayOff & Morning & Morning & Night & DayOff \\
\hline Nurse3 & Morning & Night & Afterno... & Afterno... & Night & DayOff & Morning \\
\hline Nurse3 & Afterno... & Night & Afterno... & DayOff & Night & Night & DayOff \\
\hline Nurse3 & Morning & Afterno... & Morning & DayOff & Afterno... & DayOff & Night \\
\hline Nurse4 & Night & DayOff & Afterno... & Afterno... & Night & Afterno... & Morning \\
\hline Nurse4 & Afterno... & Morning & DayOff & DayOff & Morning & Night & Afterno... \\
\hline Nurse4 & DayOff & Afterno... & Night & DayOff & DayOff & Afterno... & DayOff \\
\hline
\end{tabular}

Figure 18. Sample timetable generated using single-point crossover and boundary mutation operators.

\begin{tabular}{|c|c|c|c|c|c|c|c|}
\hline Nurse & Monday & Tuesday & Wedne... & Thursday & Friday & Saturday & Sunday \\
\hline Nurse1 & Morning & Night & Night & Night & DayOff & Afterno... & Afterno... \\
\hline Nurse1 & Night & Night & Night & DayOff & Afterno... & DayOff & DayOff \\
\hline Nurse1 & Afterno... & Morning & DayOff & Morning & Dayoff & Afterno... & Night \\
\hline Nurse1 & Afterno... & Night & Afterno... & DayOff & Afterno... & DayOff & Afterno... \\
\hline Nurse1 & Afterno... & Night & DayOff & Afterno... & DayOff & Afterno... & DayOff \\
\hline Nurse1 & Night & Afterno... & Afterno... & Morning & DayOff & Afterno... & DayOff \\
\hline Nurse2 & DayOff & Morning & Night & DayOff & Morning & Morning & DayOff \\
\hline Nurse2 & Morning & Afterno... & Afterno... & Afterno... & Morning & Morning & DayOff \\
\hline Nurse2 & DayOff & Afterno... & DayOff & Afterno... & Morning & Night & DayOff \\
\hline Nurse3 & Afterno... & Afterno... & Afterno... & Afterno... & Afterno... & DayOff & Morning \\
\hline Nurse 3 & Morning & Morning & Afterno... & DayOff & Afterno... & Night & DayOff \\
\hline Nurse 3 & Afterno... & Afterno... & Morning & Morning & Afterno... & DayOff & Night \\
\hline Nurse 4 & DayOff & Afterno... & Afterno... & Afterno... & Night & Afterno... & Morning \\
\hline Nurse4 & Afterno... & Night & DayOff & Afterno... & Morning & Night & Afterno... \\
\hline Nurse 4 & DayOff & Night & Night & DayOff & Morning & Afterno... & DayOff \\
\hline
\end{tabular}

Figure 19. Sample timetable generated using two-point crossover and uniform mutation operators. 


\begin{tabular}{|c|c|c|c|c|c|c|c|}
\hline Nurse & Monday & Tuesday & Wedne... & Thursday & Friday & Saturday & Sunday \\
\hline Nurse1 & Morning & Night & Night & Night & DayOff & Afterno... & Morning \\
\hline Nurse1 & Night & Night & Night & DayOff & Afterno... & DayOff & DayOff \\
\hline Nurse1 & Afterno... & Morning & DayOff & Morning & DayOff & Afterno... & Night \\
\hline Nurse1 & Afterno... & Night & Afterno... & DayOff & Afterno... & DayOff & Afterno... \\
\hline Nurse1 & Afterno... & DayOff & DayOff & Afterno... & DayOff & Afterno... & DayOff \\
\hline Nurse1 & Night & Afterno... & Afterno... & Morning & DayOff & Afterno... & DayOff \\
\hline Nurse2 & DayOff & Morning & Night & Dayoff & Afterno... & Morning & DayOff \\
\hline Nurse2 & Morning & Afterno... & Afterno... & Afterno... & Morning & Morning & DayOff \\
\hline Nurse2 & DayOff & Afterno... & DayOff & Afterno... & Morning & Night & DayOff \\
\hline Nurse3 & Afterno... & Afterno... & Afterno... & Afterno... & Afterno... & DayOff & Morning \\
\hline Nurse3 & Morning & Morning & Afterno... & DayOff & Afterno... & Night & DayOff \\
\hline Nurse3 & Afterno... & Afterno... & Morning & Morning & Afterno... & DayOff & Night \\
\hline Nurse4 & DayOff & Afterno... & Afterno... & Afterno... & Night & Afterno... & Morning \\
\hline Nurse4 & Afterno... & Night & DayOff & Afterno... & Morning & Night & Afterno... \\
\hline Nurse4 & DayOff & Night & Night & DayOff & Morning & Afterno... & DayOff \\
\hline
\end{tabular}

Figure 20. Sample timetable generated using two-point crossover and single mutation operators.

\begin{tabular}{|c|c|c|c|c|c|c|c|}
\hline Nurse & Monday & Tuesday & Wedne... & Thursday & Friday & Saturday & Sunday \\
\hline Nurse1 & Morning & Night & Night & Night & Dayoff & Afterno... & Morning \\
\hline Nurse1 & Night & Night & Night & DayOff & Afterno... & DayOff & CayOff \\
\hline Nurse1 & Afterno... & Morning & DayOff & Morning & DayOff & Afterno... & Night \\
\hline Nurse1 & Afterno... & Night & Afterno... & DayOff & Afterno... & DayOff & Afterno... \\
\hline Nurse1 & Afterno... & DayOff & DayOff & Afterno... & DayOff & Afterno... & CayOff \\
\hline Nurse1 & Night & Afterno... & Afterno... & Morning & DayOff & Afterno... & CayOff \\
\hline Nurse2 & DayOff & Morning & Night & DayOff & Afterno... & Morning & CayOff \\
\hline Nurse2 & Morning & Afterno... & Afterno... & Afterno... & Morning & Morning & CayOff \\
\hline Nurse2 & DayOff & Afterno... & DayOff & Afterno... & Morning & Night & CayOff \\
\hline Nurse3 & Afterno... & Afterno... & Afterno... & Night & Afterno... & DayOff & Morning \\
\hline Nurse3 & Morning & Morning & Afterno... & DayOff & Afterno... & Night & CayOff \\
\hline Nurse 3 & Afterno... & Afterno... & Morning & Morning & Afterno... & DayOff & Norning \\
\hline Nurse4 & DayOff & Afterno... & Afterno... & Afterno... & Night & Afterno... & Morning \\
\hline Nurse 4 & Afterno... & Night & DayOff & Afterno... & Morning & Night & Afterno... \\
\hline Nurse4 & DayOff & Night & Night & DayOff & Morning & Afterno... & CayOff \\
\hline
\end{tabular}

Figure 21. Sample timetable generated using two-point crossover and boundary mutation operators.

Figure 19 presents the first generation of two crossover $\left(\mathrm{C}_{\mathrm{o}} 2\right)$ and uniform mutation $\left(\mathrm{M}_{\mathrm{o}} 1\right)$ operator. $\mathrm{C}_{\mathrm{o}} 2$ performs interchanging schedules between 2 and 5 crossover points, the middle part of the timetable is inherent from the first parent while the head and tail are from the second parent. In this experiment, the uniform mutation $\left(M_{0} 1\right)$ is set to $5 \%$ of mutation rate.

Figure 20 shows the timetable of the first generation after using the two-point crossover $\left(\mathrm{C}_{\mathrm{o}} 2\right)$ and single mutation ( $\mathrm{M}_{\mathrm{o}} 2$ ) operators. The middle part of the timetable is inherited from parent 1 while the head and the tail are from parent 2 . For single mutation, the timetable shows that from night shift schedule from parent 1 , it has been mutated to afternoon shift.
Figure 21 shows the timetable obtained using twopoint crossover and boundary mutation operators. The timetable obtained after uniform crossover with 3 random points and uniform mutation at 5\% mutation rate is shown in Figure 22. Figure 23, on the other hand, shows the timetable obtained by uniform crossover with single mutation operator.

Figure 24 show a timetable produced after uniform crossover and boundary mutation is performed.

Table 4 summarizes the fitness values of the timetables obtained using the different combinations of crossover and mutation operators of the genetic algorithm after evaluating 1000 generations of the timetables. The highest fitness value is then considered as the best timetable. As 


\begin{tabular}{|c|c|c|c|c|c|c|c|}
\hline Nurse & Monđay & Tuesday & wedne... & Thursday & Friday & Saturday & Sunday \\
\hline Nurse1 & Afterno... & Morning & DayOff & Moming & DayOff & Afterno... & Morning \\
\hline Nurse1 & Morning & Afterno... & Afterno... & Afterno... & Afterno... & DayOff & DayOff \\
\hline Nurse1 & Afterno... & Night & DayOff & Night & DayOff & Afterno... & Night \\
\hline Nurse1 & DayOff & Night & Afterno... & Night & Afterno... & DayOff & Afterno... \\
\hline Nurse1 & Afterno... & Afterno... & Night & DayOff & DayOff & Afterno... & DayOff \\
\hline Nurse1 & Morning & Morning & Afterno... & Afterno... & DayOff & Afterno... & DayOff \\
\hline Nurse2 & Night & DayOff & Night & Afterno... & Afterno... & Morning & DayOff \\
\hline Nurse2 & Dayoff & DayOff & DayOff & Night & DayOff & Morning & DayOff \\
\hline Nurse2 & Morning & Morning & DayOff & Morning & Morning & Night & DayOff \\
\hline Nurse3 & Morning & Night & DayOff & Afterno... & Afterno... & DayOff & Morning \\
\hline Nurse3 & Afterno... & Night & Afterno... & DayOff & Afterno... & Night & DayOff \\
\hline Nurse3 & Morning & Afterno... & Night & DayOff & Afterno... & DayOff & Night \\
\hline Nurse4 & Night & DayOff & Morning & Afterno... & Night & Afterno... & Morning \\
\hline Nurse4 & Afterno... & Morning & Afterno... & DayOff & Morning & Night & Afterno... \\
\hline Nurse4 & DayOff & Afterno... & Afterno... & DayOff & Morning & Afterno... & DayOff \\
\hline
\end{tabular}

Figure 22. Sample timetable generated using uniform crossover and uniform mutation operators.

\begin{tabular}{|l|lll|ll|l|l|l|}
\hline Nurse & Monday & Tuesday & Wedne... & \multicolumn{2}{l|}{ Thursday } & Friday & \multicolumn{1}{|l|}{ Saturday } & Sunday \\
Nurse1 & Afterno... & Morning & DayOff & Morning & DayOff & Afterno... & Morning \\
Nurse1 & Morning & Afterno... & Afterno... & Afterno... & Afterno... & DayOff & DayOff \\
Nurse1 & Afterno... & Night & DayOff & Afterno... & DayOff & Afterno... & Night \\
Nurse1 & DayOff & Night & Afterno... & Night & Afterno... & DayOff & Afterno... \\
Nurse1 & Afterno... & Afterno... & Night & DayOff & DayOff & Afterno... & DayOff \\
Nurse1 & Morning & Morning & Morning & Afterno... & DayOff & Afterno... & DayOff \\
Nurse2 & Night & DayOff & Night & Afterno... & Afterno... & Morning & DayOff \\
Nurse2 & DayOff & DayOff & DayOff & Night & DayOff & Morning & DayOff \\
Nurse2 & Morning & Morning & DayOff & Morning & Morning & Night & DayOff \\
Nurse3 & Morning & Night & DayOff & Afterno... & Afterno... & DayOff & Morning \\
Nurse3 & Afterno... & Night & Afterno... & DayOff & Afterno... & Night & DayOff \\
Nurse3 & Morning & Afterno... & Night & DayOff & Afterno... & DayOff & Night \\
Nurse4 & Night & DayOff & Morning & Afterno... & Night & Afterno... & Morning \\
Nurse4 & Afterno... & Morning & Afterno... & DayOff & Morning & Night & Afterno... \\
Nurse4 & DayOff & Afterno... & Afterno... & DayOff & Morning & Afterno... & DayOff \\
\hline
\end{tabular}

Figure 23. Sample timetable generated using uniform crossover and single mutation operators.

\begin{tabular}{|c|c|c|c|c|c|c|c|}
\hline Nurse & Monday & Tuesday & Wedne... & Thursday & Friday & Saturday & Sunday \\
\hline Nurse 1 & Afterno... & Morning & DayOff & Morning & DayOff & Afterno... & Morning \\
\hline Nurse1 & Morning & Afterno... & Afterno... & Afterno... & Afterno... & DayOff & DayOff \\
\hline Nurse1 & Afterno... & Night & DayOff & Afterno... & DayOff & Afterno... & Night \\
\hline Nurse1 & DayOff & Night & Afterno... & Night & Afterno... & DayOff & Afterno... \\
\hline Nurse 1 & Afterno... & Afterno... & Night & Dayoff & DayOff & Afterno... & DayOff \\
\hline Nurse1 & Morning & Morning & Morning & Afterno... & DayOff & Afterno... & DayOff \\
\hline Nurse 2 & Night & Dayoff & Night & Afterno... & Afterno... & Morning & DayOff \\
\hline Nurse 2 & DayOff & Dayoff & DayOff & Morning & Morning & Morning & DayOff \\
\hline Nurse 2 & Morning & Morning & DayOff & Morning & Morning & Night & DayOff \\
\hline Nurse3 & Morning & Night & DayOff & Afterno... & Afterno... & DayOff & Morning \\
\hline Nurse 3 & Afterno... & Night & Afterno... & DayOff & Afterno... & Night & DayOff \\
\hline Nurse 3 & Morning & Afterno... & Night & DayOff & Afterno... & DayOff & Night \\
\hline Nurse 4 & Night & Dayoff & Morning & Afterno... & Night & Afterno... & Morning \\
\hline Nurse 4 & Afterno... & Morning & Afterno... & DayOff & Morning & Night & Afterno... \\
\hline Nurse 4 & DayOff & Afterno... & Afterno... & Dayoff & Morning & Afterno... & DayOff \\
\hline
\end{tabular}

Figure 24. Sample timetable generated using uniform crossover and boundary mutation operators. 
Table 4. Summary of fitness values of the different genetic operators after 1000 generations

\begin{tabular}{|c|c|c|c|c|c|c|c|c|c|}
\hline \multirow{2}{*}{$\begin{array}{l}\text { No. of } \\
\text { Generations }\end{array}$} & \multicolumn{9}{|c|}{ Genetic Operators } \\
\hline & $\mathrm{C}_{\mathrm{o}} 1$ and $\mathrm{M}_{\mathrm{o}} 1$ & $\begin{array}{c}\mathrm{C}_{\mathrm{o}} 1 \text { and } \\
\mathrm{M}_{0} 2\end{array}$ & $\begin{array}{c}\mathrm{C}_{\mathrm{o}} 1 \text { and } \\
\mathrm{M}_{0} 3\end{array}$ & $\mathrm{C}_{\mathrm{o}} 2$ and $\mathrm{M}_{\mathrm{o}} 1$ & $\begin{array}{c}\mathrm{C}_{\mathrm{o}} 2 \text { and } \\
\mathrm{M}_{0} 2\end{array}$ & $\begin{array}{c}\mathrm{C}_{\mathrm{o}} 2 \text { and } \\
\mathrm{M}_{0} 3\end{array}$ & $\begin{array}{c}C_{0} 3 \text { and } \\
M_{0} 1\end{array}$ & $\begin{array}{c}\mathrm{C}_{\mathrm{o}} 3 \text { and } \\
\mathrm{M}_{0} 2\end{array}$ & $\begin{array}{c}\mathrm{C}_{\mathrm{o}} 3 \text { and } \\
\mathrm{M}_{0} 3\end{array}$ \\
\hline 50 & 0.0263 & 0.0278 & 0.0200 & 0.0278 & 0.0250 & 0.0217 & 0.0250 & 0.0222 & 0.0217 \\
\hline 100 & 0.0323 & 0.0303 & 0.0213 & 0.0333 & 0.0303 & 0.0222 & 0.0294 & 0.0286 & 0.0244 \\
\hline 150 & 0.0400 & 0.0322 & 0.0213 & 0.0385 & 0.0385 & 0.0222 & 0.0384 & 0.0322 & 0.0244 \\
\hline 200 & 0.0417 & 0.0357 & 0.0213 & 0.0417 & 0.0400 & 0.0222 & 0.0416 & 0.0385 & 0.0244 \\
\hline 250 & 0.0435 & 0.0400 & 0.0213 & 0.04348 & 0.0435 & 0.0222 & 0.0434 & 0.0385 & 0.0244 \\
\hline 300 & 0.0435 & 0.0435 & 0.0213 & 0.04348 & 0.0476 & 0.0222 & 0.0434 & 0.0385 & 0.0244 \\
\hline 350 & 0.0435 & 0.0435 & 0.0213 & 0.04348 & 0.0476 & 0.0222 & 0.0500 & 0.0385 & 0.0244 \\
\hline 400 & 0.0476 & 0.0454 & 0.0213 & 0.04545 & 0.0500 & 0.0222 & 0.0526 & 0.0400 & 0.0244 \\
\hline 450 & 0.0500 & 0.0454 & 0.0213 & 0.04545 & 0.0500 & 0.0222 & 0.0556 & 0.0400 & 0.0244 \\
\hline 500 & 0.0500 & 0.0454 & 0.0213 & 0.04545 & 0.0526 & 0.0222 & 0.0588 & 0.0455 & 0.0244 \\
\hline 550 & 0.0500 & 0.0454 & 0.0213 & 0.04545 & 0.0556 & 0.0222 & 0.0588 & 0.0455 & 0.0244 \\
\hline 600 & 0.0500 & 0.0454 & 0.0213 & 0.04762 & 0.0556 & 0.0222 & 0.0588 & 0.0500 & 0.0244 \\
\hline 650 & 0.0500 & 0.0454 & 0.0213 & 0.05000 & 0.0556 & 0.0222 & 0.0625 & 0.0500 & 0.0244 \\
\hline 700 & 0.0500 & 0.0476 & 0.0213 & 0.05000 & 0.0556 & 0.0222 & 0.0625 & 0.0500 & 0.0244 \\
\hline 750 & 0.0556 & 0.0500 & 0.0213 & 0.05000 & 0.0625 & 0.0222 & 0.0625 & 0.0500 & 0.0244 \\
\hline 800 & 0.0556 & 0.0526 & 0.0213 & 0.05000 & 0.0625 & 0.0222 & 0.0625 & 0.0500 & 0.0244 \\
\hline 850 & 0.0556 & 0.0526 & 0.0213 & 0.0588 & 0.0667 & 0.0222 & 0.0625 & 0.0500 & 0.0244 \\
\hline 900 & 0.0556 & 0.0526 & 0.0213 & 0.0588 & 0.0667 & 0.0222 & 0.0625 & 0.0500 & 0.0244 \\
\hline 950 & 0.0588 & 0.0526 & 0.0213 & 0.0588 & 0.0667 & 0.0222 & 0.0625 & 0.0500 & 0.0244 \\
\hline 1000 & 0.0588 & 0.0526 & 0.0213 & 0.0588 & 0.0667 & 0.0222 & 0.0625 & 0.0500 & 0.0244 \\
\hline
\end{tabular}

shown in the table, among the three parameters of genetic algorithm with $C_{o} 1$ as crossover operator, the pair $C_{o} 1$ and $M_{o} 1$ performs best in producing timetables for nurse schedule as it has a fitness value of 0.0588 . On the other hand, the pair $C_{o} 2$ and $M_{o} 2$ has the highest fitness value for the two-point crossover with a fitness value of 0.0667 while the pair $C_{o} 3$ and $M_{o} 1$ gives the best timetable for the uniform crossover operator with a fitness value of 0.0625 .

Among the 9 combinations considered, the highest fitness value is that of the pair $C_{o} 2$ and $M_{o} 2$, that is, twopoint crossover and single mutation operators, with a fitness value of 0.0667 . The timetable produced using this combination is considered as the best timetable for the nurse scheduling problem. This timetable is shown in Figure 25. It can be seen that there are 14 violations of the soft constraints in this timetable, namely, 2 counts of violations of soft constraint \#1, 1 for soft constraint \#2, 4 for soft constraint \#3 and 7 violations for soft constraint \#6. However, it can be observed that the shifts have been properly distributed among the nurses. Although there are few violations committed, the assignments of the shifts are satisfied.
It can also be seen from Table 4 that the timetable obtained using the pair $C_{o} 1$ and $\mathrm{M}_{\mathrm{o}} 3$, that is, the pair single point crossover and boundary mutation operators, has the smallest fitness value of 0.0213 . This timetable is shown in Figure 26. It can be seen that this timetable has 46 violations of the soft constraints. These includes 8 counts of violations of soft constraint \#1, i.e., every nurse is assigned to more than 2 days in every shift, 1 count of violation soft constraint \#2, i.e., third nurse level 4 has not been assigned to a night shift, 11 counts of violations of soft constraint \#3 are observed and 8 violations of soft constraint \#4, 10 violations of soft constraint \#5, i.e. there are levels 2, 3, 4 nurses assigned on Sundays, and 8 violations of soft constraint \#6, i.e. level 3 and 4 nurses work on night shift are also observed. It can also be seen that the shifts in this timetable are not properly distributed as there are nurses assigned to more than 1 day off while other nurses do not have days off at all. It can also be observed that some nurses are assigned to more than two days in each shift and other level 3 and 4 nurses assigned to night shift. 


\begin{tabular}{l|l|l|l|l|l|l|l|} 
Nurse & Monday & Tuesday & Wedne... & Thursday & Friday & Saturday & Sunday \\
\hline Nurse1 & DayOff & Afterno... & Morning & Night & Night & Afterno... & Morning \\
\hline Nurse1 & DayOff & Morning & Afterno... & Night & Night & Afterno... & Morning \\
\hline Nurse1 & Afterno... & Afterno... & Night & DayOff & Morning & Morning & Night \\
\hline Nurse1 & Night & Afterno... & Morning & Afterno... & Night & DayOff & Morning \\
\hline Nurse1 & Morning & Afterno... & Night & Night & Afterno... & Morning & DayOff \\
\hline Nurse1 & Morning & Morning & Afterno... & Afterno... & Night & Night & DayOff \\
\hline Nurse2 & Morning & Afterno... & DayOff & Morning & Night & Night & Afterno... \\
\hline Nurse2 & Night & Afterno... & Night & Afterno... & Morning & Morning & DayOff \\
\hline Nurse2 & Morning & Afterno... & Morning & Night & Night & Afterno... & DayOff \\
\hline Nurse3 & Afterno... & Morning & DayOff & Morning & Afterno... & Night & DayOff \\
\hline Nurse3 & Morning & Afterno... & Night & Afterno... & Morning & Afterno... & DayOff \\
\hline Nurse3 & Morning & Morning & DayOff & Night & Afterno... & Afterno... & DayOff \\
\hline Nurse4 & Morning & Afterno... & Morning & Afterno... & Afterno... & Night & DayOff \\
\hline Nurse4 & Morning & Night & Afterno... & DayOff & Morning & Afterno... & DayOff \\
\hline Nurse4 & Morning & Afterno... & Afterno... & Morning & DayOff & DayOff & DayOff \\
\hline
\end{tabular}

Figure 25. Timetable produced by two-point crossover and single mutation operator after 1000 generations.

\begin{tabular}{l|l|l|l|l|l|l|l|} 
Nurse & Monday & Tuesday & Wedne... & Thursday & Friday & Saturday & Sunday \\
\hline Nurse1 & DayOff & Afterno... & Morning & Afterno... & Night & Afterno... & DayOff \\
\hline Nurse1 & DayOff & DayOff & Afterno... & DayOff & Night & Afterno... & Morning \\
\hline Nurse1 & Afterno... & Night & Night & DayOff & Morning & Morning & Night \\
\hline Nurse1 & Night & Afterno... & Afterno... & Afterno... & Night & DayOff & Morning \\
\hline Nurse1 & Night & Afterno... & Night & Afterno... & Afterno... & Morning & Afterno... \\
\hline Nurse1 & Morning & Morning & Afterno... & Afterno... & DayOff & Night & DayOff \\
\hline Nurse2 & Afterno... & Afterno... & DayOff & Morning & Morning & Night & Night \\
\hline Nurse2 & Night & Afterno... & Night & Afterno... & Morning & DayOff & DayOff \\
\hline Nurse2 & Morning & DayOff & Afterno... & Morning & Night & Afterno... & DayOff \\
\hline Nurse3 & Night & DayOff & DayOff & Morning & Afterno... & Morning & Afterno... \\
\hline Nurse3 & Afterno... & Afterno... & Night & Afterno... & Morning & Afterno... & Morning \\
\hline Nurse3 & Afterno... & Morning & Morning & Night & Night & DayOff & Afterno... \\
\hline Nurse4 & Morning & Afterno... & Morning & Afterno... & Afterno... & Night & DayOff \\
\hline Nurse4 & Dayoff & Night & Dayoff & DayOff & Morning & Afterno... & Morning \\
\hline Nurse4 & Morning & Morning & Afterno... & Afterno... & DayOff & DayOff & DayOff \\
\hline
\end{tabular}

Figure 26. Timetable produced by single point crossover and boundary mutation operator after 1000 generations.

Figure 27 shows how the fitness values of the timetables obtained using the various combinations of crossover and mutation operators behave during the 1000 generations.
It can be seen that the pair $\mathrm{C}_{0} 2$ and $\mathrm{M}_{\mathrm{o}} 2$ gives the highest fitness value while the pair $C_{o} 1$ and $M_{o} 3$ gives the lowest. 


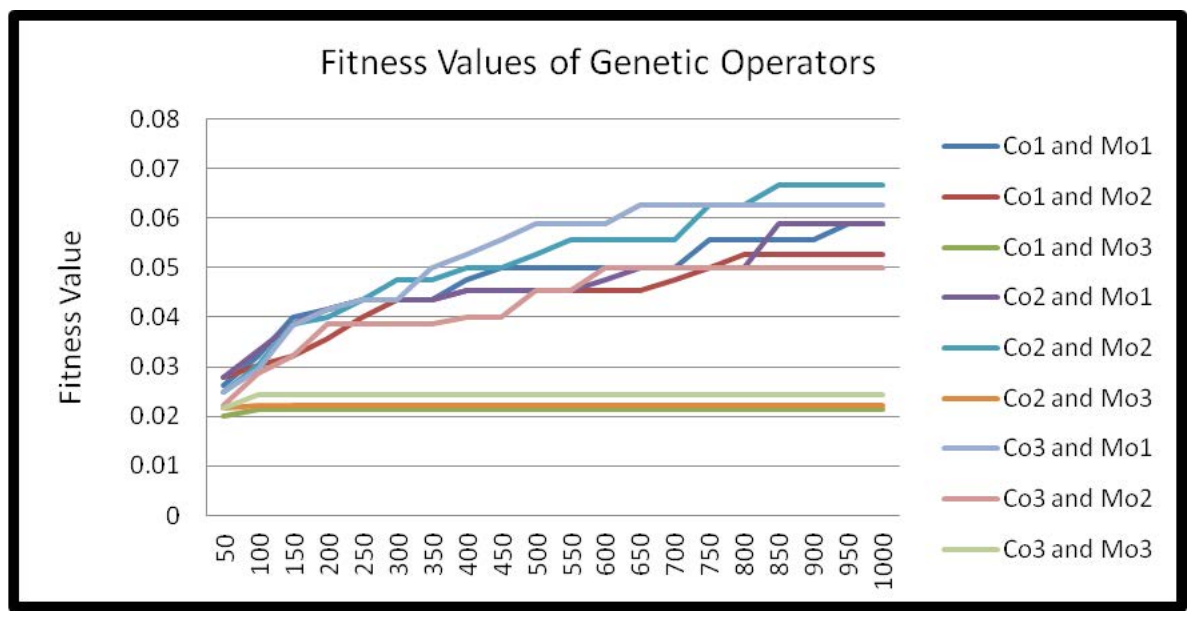

Figure 27. Fitness values of different genetic operators after 1000 generations.

Table 5. Summary of total salary cost (in PHP) for each timetable after 1000 generations

\begin{tabular}{|c|c|c|c|c|c|c|c|c|c|}
\hline \multirow{2}{*}{$\begin{array}{l}\text { No. of } \\
\text { Generations }\end{array}$} & \multicolumn{9}{|c|}{ Genetic Operators } \\
\hline & $\begin{array}{c}\mathrm{C}_{\mathrm{o}} 1 \text { and } \\
\mathrm{M}_{0} 1\end{array}$ & $\begin{array}{c}\mathrm{C}_{\mathrm{o}} 1 \text { and } \\
\mathrm{M}_{0} 2\end{array}$ & $\begin{array}{c}\mathrm{C}_{\mathrm{o}} 1 \text { and } \\
\mathrm{M}_{0} 3\end{array}$ & $\begin{array}{c}\mathrm{C}_{\mathrm{o}} 2 \text { and } \\
\mathrm{M}_{0} 1\end{array}$ & $\begin{array}{c}\mathrm{C}_{\mathrm{o}} 2 \text { and } \\
\mathrm{M}_{0} 2\end{array}$ & $\begin{array}{c}\mathrm{C}_{\mathrm{o}} 2 \text { and } \\
\mathrm{M}_{0} 3\end{array}$ & $\begin{array}{c}C_{0} 3 \text { and } \\
M_{0} 1\end{array}$ & $\begin{array}{c}\mathrm{C}_{\mathrm{o}} 3 \text { and } \\
\mathrm{M}_{0} 2\end{array}$ & $\begin{array}{c}\mathrm{C}_{\mathrm{o}} 3 \text { and } \\
\mathrm{M}_{0} 3\end{array}$ \\
\hline 50 & 24,038 & 22,273 & 28,286 & 23,129 & 24,610 & 29,733 & 24,065 & 26,261 & 27,976 \\
\hline 100 & 19,349 & 21,114 & 27,630 & 18,830 & 20,535 & 28,073 & 21,340 & 22,172 & 26,516 \\
\hline 150 & 14,411 & 19,962 & 27,630 & 16,633 & 16,338 & 28,073 & 15,203 & 19,627 & 26,516 \\
\hline 200 & 14,155 & 17,704 & 27,630 & 14,315 & 15,877 & 28,073 & 14,212 & 17,515 & 26,516 \\
\hline 250 & 13,317 & 16,544 & 27,630 & 13,785 & 14,751 & 28,073 & 13,790 & 17,515 & 26,516 \\
\hline 300 & 13,317 & 14,364 & 27,630 & 13,785 & 13,675 & 28,073 & 13,790 & 17,515 & 26,516 \\
\hline 350 & 12,856 & 14,364 & 27,630 & 13,785 & 13,675 & 28,073 & 12,369 & 17,515 & 26,516 \\
\hline 400 & 12,434 & 13,411 & 27,630 & 13,093 & 12,753 & 28,073 & 11,544 & 17,131 & 26,516 \\
\hline 450 & 11,888 & 13,411 & 27,630 & 13,093 & 12,753 & 28,073 & 11,075 & 17,131 & 26,516 \\
\hline 500 & 11,888 & 13,411 & 27,630 & 13,093 & 12,177 & 28,073 & 10,661 & 15,249 & 26,516 \\
\hline 550 & 11,888 & 13,411 & 27,630 & 13,093 & 11,716 & 28,073 & 10,661 & 15,249 & 26,516 \\
\hline 600 & 11,888 & 13,411 & 27,630 & 12,748 & 11,716 & 28,073 & 10,661 & 12,427 & 26,516 \\
\hline 650 & 11,888 & 13,411 & 27,630 & 12,748 & 11,716 & 28,073 & 10,095 & 12,427 & 26,516 \\
\hline 700 & 11,888 & 12,970 & 27,630 & 12,748 & 11,716 & 28,073 & 10,095 & 12,427 & 26,516 \\
\hline 750 & 10,776 & 12,566 & 27,630 & 12,748 & 9,823 & 28,073 & 10,095 & 12,427 & 26,516 \\
\hline 800 & 10,776 & 12,182 & 27,630 & 12,748 & 9,823 & 28,073 & 10,095 & 12,427 & 26,516 \\
\hline 850 & 10,776 & 12,182 & 27,630 & 10,481 & 9,624 & 28,073 & 10,095 & 12,427 & 26,516 \\
\hline 900 & 10,776 & 12,182 & 27,630 & 10,481 & 9,624 & 28,073 & 10,095 & 12,427 & 26,516 \\
\hline 950 & 10,776 & 12,182 & 27,630 & 10,481 & 9,624 & 28,073 & 10,095 & 12,427 & 26,516 \\
\hline 1000 & 10,776 & 12,182 & 27,630 & 10,481 & 9,624 & 28,073 & 10,095 & 12,427 & 26,516 \\
\hline
\end{tabular}

The timetable obtained after 1000 generations using the different combinations of genetic operators have also been evaluated in terms of the objective function value, that is, the total salary cost. A summary of the total salary cost for each timetable is shown in Table 5. It can be seen from the table that the timetable obtained using the combination two-point crossover and single mutation operators give the smallest total salary cost in the amount of PHP 9624. 


\section{Conclusions}

In this study, a genetic algorithm is used to solve nurse scheduling problem for private hospitals in the Philippines. This study considered preferences of nurses in terms of work schedule while meeting the objective of the hospital management of minimizing total salary cost. Among the operators tested, the pair two-point crossover and single mutation an operator outperforms than other genetic operators in terms of fitness value and total salary cost of its resulting timetable.

This paper worked with operators for the genetic algorithm in solving the NSP. It might be interesting to consider a similar study involving GA and other metaheuristic approaches in solving the university course scheduling system 12 .

\section{References}

1. Miller H, Pierskalla W, Rath G. Nurse scheduling using mathematical programming. Operations. 1976; 24(5):85770 .

2. Brigitte J, Semet F, Vovor T. A generalized linear programming model for nurse scheduling. European Journal of Operational Research. 1998; 107(1):1-18.

3. Aickelin U, Dowsland KA. Exploiting problem structure in a genetic algorithm approach to a nurse rostering problem. Journal of Scheduling. 2000; 3(1):139-53.

4. Burke E, Cowling P, Causmaecker P, Berghe GV. A memetic approach to the nurse rostering problem. Applied Intelligence. 2001; 15(3):199-214.

5. Ikegami A, Niwa A. A subproblem-centric model and approach to the nurse scheduling problem. Mathematical Programming. 2003; 97(3):517-41.

6. Maenhout B, Vanhoucke M. Comparison and hybridization of crossover operators for the nurse scheduling problem. Annals of Operations Research. 2007; 159:333-53.

7. Tsai CC, Lee CJ. Optimization of nurse scheduling problem with a two-stage mathematical programming model. Asia Pacific Management Review. 2010; 15(4):503-16.

8. Moz M, Pato MV. Genetic algorithm approach to a nurse rerostering problem. Computers and Operations Research. 2007; 34(3):667-91.

9. Davis L. Handbook of Genetic Algorithms, 1991. Van NostrandReinhol: New York, USA; 1991.

10. Beasley D, Bull D, Martin R. An overview of genetic algorithms: Part 1, Fundamentals. University Computing. 1993; 15(2):58-69.

11. Leksakul K, Phetsawat $\mathrm{S}$. Nurse scheduling using genetic algorithm. Mathematical Problems in Engineering. 2014; 2014.

12. Rohini V, Natarajan AM. Comparison of genetic algorithm with Particle Swarm Optimisation, ant Colony Optimisationand Tabusearch based on university course scheduling system. Indian Journal of Science and Technology. 2016; 9(21):1-5. 